\title{
Bevacizumab treatment of meningeal melanoma metastases
}

\author{
Trude G. Simonsen, Jon-Vidar Gaustad and Einar K. Rofstad ${ }^{*}$
}

\begin{abstract}
Background: Melanoma patients with metastatic growth in the meninges have poor prognosis and few treatment options. Although treatment with BRAF inhibitors or immune checkpoint inhibitors has provided promising results, most patients with advanced melanoma are resistant to these treatments and develop severe side effects. Novel treatment strategies are needed for patients with meningeal melanoma metastases, and the potential of antiangiogenic therapy was investigated in this preclinical study.

Methods: Two GFP-transfected melanoma models (A-07 and D-12) differing substantially in VEGF-A expression were included in the study, and the anti-VEGF-A antibody bevacizumab was used as therapeutic agent. Meningeal metastases were initiated in BALB/c nu/nu mice by intracranial inoculation of melanoma cells, and bevacizumab treatment was given twice a week in i.p. doses of $10 \mathrm{mg} / \mathrm{kg}$ until the mice became moribund. Therapeutic effects were evaluated by determining tumor host survival time, assessing tumor growth and angiogenic activity by quantitative analyses of histological preparations, and measuring the expression of angiogenesis-related genes by quantitative PCR.

Results: Meningeal A-07 melanomas showed higher expression of VEGF-A than meningeal D-12 melanomas, whereas the expression of ANGPT2 and IL8, two important angiogenesis drivers in melanoma, was much higher in D-12 than in A-07 tumors. Bevacizumab treatment inhibited tumor angiogenesis and prolonged host survival in mice with A-07 tumors but not in mice with D-12 tumors. Meningeal A-07 tumors in bevacizumab-treated mice compensated for the reduced VEGF-A activity by up-regulating a large number of angiogenesis-related genes, including ANGPT2 and its receptors TIE1 and TIE2. Melanoma cells migrated from meningeal tumors into the cerebrum, where they initiated metastatic growth by vessel co-option. In the A-07 model, the density of cerebral micrometastases was higher in bevacizumab-treated than in untreated mice, either because bevacizumab treatment increased mouse survival or induced increased tumor gene expression.
\end{abstract}

Conclusions: The development of antiangiogenic strategies for the treatment of meningeal melanoma metastases is a challenging task because the outcome of treatment will depend on the angiogenic signature of the tumor tissue, treatment-induced alterations of the angiogenic signature, and the treatment sensitivity of metastatic lesions in other intracranial sites.

Keywords: Melanoma, Meningeal metastasis, Antiangiogenic therapy, Bevacizumab, VEGF

${ }^{*}$ Correspondence: einar.k.rofstad@rr-research.no

Group of Radiation Biology and Tumor Physiology, Department of Radiation Biology, Institute for Cancer Research, Oslo University Hospital, Oslo, Norway

\section{Background}

Patients diagnosed with malignant melanoma have a high risk of developing brain metastases [1]. Approximately $50 \%$ of the patients with advanced-stage disease show brain involvement during the course of their disease, and this incidence has increased to $\sim 75 \%$ at autopsy [2-4]. The prognosis of the patients with brain involvement is 
poor, with a median overall survival of 4-6 months [2, $5,6]$. A majority of these patients show multiple brain lesions and/or meningeal involvement, and these factors are associated with particularly poor prognosis [2, 5]. While current treatment options for patients with a limited number of brain parenchymal lesions include surgical resection and stereotactic radiosurgery, the treatment options for patients with multifocal disease and meningeal involvement are mainly palliative and include whole-brain radiotherapy and chemotherapy $[1,7,8]$. Recently, the introduction of molecularly targeted therapies and immunotherapy has given hope to patients with advanced melanoma $[9,10]$, and several trials are evaluating the effect of BRAF inhibitors and immune checkpoint inhibitors in patients with brain metastases $[1,11]$. Although initial results are encouraging, a substantial proportion of the patients show intrinsic or acquired treatment resistance, and the drugs are also associated with severe side effects $[9,11]$. Consequently, it is important to explore further treatment strategies to improve the dismal prognosis of this patient group.

The high incidence of brain involvement in autopsy studies suggests that subclinical brain lesions are common in melanoma patients [3, 12]. With prolonged patient survival due to improved control of extracranial disease, these subclinical lesions may be allowed more time to develop and grow into symptomatic brain metastases [13]. Brain metastases cause deteriorating and sometimes irreversible neurological symptoms, and a strategy to prevent the growth of brain metastases would be of great clinical benefit [13]. It is well established that melanoma growth and progression depend on angiogenesis, and vascular endothelial growth factor A (VEGF-A) has been shown to be an important angiogenic driver in melanoma [14]. Antiangiogenic therapy targeting the VEGF-A pathway has thus been suggested as a possible strategy to prevent melanoma relapse and spread after locoregional surgery, and adjuvant treatment with the anti-VEGF-A antibody bevacizumab [15] is currently being investigated in a large multicenter randomized phase 3 trial in melanoma patients with high risk of recurrence [16]. Furthermore, high expression of VEGF-A has been associated with the vascularization and growth of melanoma brain metastases in preclinical studies [17-20], and a recent study including both clinical and preclinical experiments suggests that bevacizumab can prevent the formation of brain metastases in lung adenocarcinoma [21]. Antiangiogenic therapy targeting the VEGF-A pathway may thus represent a potential strategy to prevent metastasis to the brain in melanoma patients.

Therapeutic effects of antiangiogenic agents in melanoma brain metastases have been examined in a few preclinical studies [22-24], and these studies have provided varying results, possibly reflecting a complex role of angiogenesis and VEGF-A in the formation and growth of metastases in the brain [1]. The vascularization of melanoma brain metastases may occur by sprouting angiogenesis (i.e., the sprouting of new vessels from pre-existing vessels) as well as vessel co-option (i.e., the growth of tumor cells along pre-existing vessels without the formation of new vessels) $[19,23,25,26]$. Although high VEGF-A expression is generally associated with sprouting angiogenesis [19], vessel co-option has also been observed in melanoma models with high VEGF-A expression [27], and VEGF-A has been shown to induce progression of experimental melanoma brain metastases without inducing sprouting angiogenesis [17]. Moreover, different VEGF-A isoforms may have differential effects on the development and growth of metastatic disease in the brain [18].

Melanomas may form metastases in multiple intracranial sites, and these metastases may differ in vascularization and response to antiangiogenic agents [23, 24, 27]. Meningeal metastases show signal enhancement in contrast-enhanced MRI, suggesting leaky vessels and sprouting angiogenesis [8]. Furthermore, the level of VEGF-A in the cerebrospinal fluid has been shown to have diagnostic and prognostic value [28]. These observations suggest that meningeal lesions may be susceptible to antiangiogenic agents targeting the VEGF-A pathway. This possibility was investigated in the study reported in the present communication. Two preclinical models of meningeal melanoma metastases differing highly in VEGF-A expression were included in the study, and bevacizumab was used as therapeutic agent. Effects of treatment were evaluated by assessing tumor host survival, analyzing tumor growth and vascularity in histological preparations, and measuring the expression of angiogenesis-related genes.

\section{Materials and methods \\ Mice}

Adult (8-10 weeks of age) female BALB/c nu/nu mice were used as host animals. The mice were bred at our institute and maintained under specific pathogen-free conditions at a temperature of $22-24{ }^{\circ} \mathrm{C}$ and a humidity of $30-50 \%$. The animal experiments were approved by the Institutional Committee on Research Animal Care, Department of Comparative Medicine, Oslo University Hospital, Norway and the Norwegian Food Safety Authority, Brumunddal, Norway (Approval: FOTS ID 10422), and were performed in accordance with the Interdisciplinary Principles and Guidelines for the Use of Animals in Research, Marketing, and Education (New York Academy of Sciences, New York, NY) and the EU 
Directive 2010/63/EU for animal experiments (http:// ec.europa.eu/environment/chemicals/lab_animals/legis lation_en.htm).

\section{Cell lines}

A-07 and D-12 human melanoma cells [29] constitutively transfected with green fluorescence protein (GFP) were obtained from our frozen stock and maintained as monolayers in RPMI 1640 (25 mM HEPES and L-glutamine) medium supplemented with $13 \%$ bovine calf serum, $250 \mu \mathrm{g} / \mathrm{mL}$ penicillin, $50 \mu \mathrm{g} / \mathrm{mL}$ streptomycin, and $700 \mu \mathrm{g} / \mathrm{mL}$ (A-07) or $900 \mu \mathrm{g} / \mathrm{mL}$ (D-12) genetecin. The cultures were incubated at $37{ }^{\circ} \mathrm{C}$ in a humidified atmosphere of $5 \% \mathrm{CO}_{2}$ in air and subcultured twice a week. Cells were harvested from exponentially growing cultures and resuspended in $\mathrm{Ca}^{2+}$-free and $\mathrm{Mg}^{2+}$-free Hanks' balanced salt solution (HBSS) before injection into animals.

\section{Anesthesia}

Intracranial injection of tumor cells was carried out with anesthetized mice. Fentanyl citrate (Janssen Pharmaceutica, Beerse, Belgium), fluanisone (Janssen Pharmaceutica), and midazolam (Hoffmann-La Roche, Basel, Switzerland) were administered intraperitoneally (i.p.) in doses of $0.63 \mathrm{mg} / \mathrm{kg}, 20 \mathrm{mg} / \mathrm{kg}$, and $10 \mathrm{mg} / \mathrm{kg}$, respectively. The body core temperature of the mice was maintained at $37-38^{\circ} \mathrm{C}$ by using a heating pad.

\section{Intracranial tumor cell injection}

The mice were fixed in a stereotactic apparatus (Model 900; Kopf Instruments, Tujunga, CA) for injection of tumor cells into the right hemisphere. The injection point was $2 \mathrm{~mm}$ anterior to the coronal and $1 \mathrm{~mm}$ lateral to the sagittal suture lines. A $100-\mu \mathrm{L}$ Hamilton syringe with a 26 -gauge needle was used to inject $3.0 \times 10^{3}$ cells suspended in $6 \mu \mathrm{L}$ of HBSS. To minimize cell reflux, the cells were injected slowly and the needle was left in place for 2 min before it was retracted slowly.

\section{Bevacizumab treatment}

Bevacizumab (Avastin; Hoffman-La Roche, Basel, Switzerland) was dissolved in physiological saline and administered i.p. in doses of $10 \mathrm{mg} / \mathrm{kg}$. Treatment with bevacizumab or vehicle was initiated 1 day before tumor cell injection, and continued twice a week until the mice became moribund. The mice were examined daily for clinical signs of tumor growth. Moribund mice were killed and autopsied, and the brain was removed for subsequent histological analysis or quantitative PCR of the tumor tissue. The survival time of the mice was calculated as the time from tumor cell injection until the mice became moribund and were euthanized.

\section{Histological analysis}

The brain was fixed in phosphate-buffered $4 \%$ paraformaldehyde and embedded in paraffin. Histological sections were cut and stained with hematoxylin and eosin (HE) using standard procedures or immunostained by using a peroxidase-based indirect staining method [30]. An anti-GFP rabbit polyclonal antibody (Abcam, Cambridge, UK) or an anti-CD31 rabbit polyclonal antibody (Abcam) was used as primary antibody to detect melanoma cells or endothelial cells, respectively. Diaminobenzidine was used as chromogen, and counterstaining was carried out with hematoxylin. Microvascular density (MVD) was scored by counting CD31-positive vessels, and tumor angiogenic activity (i.e., the rate of generation of blood vessels) was calculated from the tumor cross-section, MVD, and the time from initiation of angiogenesis to tumor removal [31]. Micrometastases within the cerebral parenchyma were scored by counting colonies of GFP-positive tumor cells. Three brain sections from each mouse were subjected to quantitative analysis.

\section{Quantitative PCR}

The $\mathrm{RT}^{2}$ Profiler PCR Array Human Angiogenesis (PAHS-024Z; SABiosciences, Frederick, MD) was used for expression profiling of angiogenesis-related genes. Total RNA was isolated from cultured cells in exponential growth or from tumor tissue stabilized in RNAlater RNA Stabilization Reagent (Qiagen, Hilden, Germany). RNA isolation, cDNA synthesis, and real-time PCR were performed as described in detail previously [32]. Fold difference in gene expression was calculated by using the $\Delta \Delta \mathrm{C}_{\mathrm{T}}$-method [33]. A $\mathrm{C}_{\mathrm{T}}$-value of 35 (15 cycles above the positive PCR control) was set as detection limit. The array included 5 housekeeping genes $[\beta$-actin (ACTB), $\beta$-2-microglobulin (B2M), glyceraldehyde-3-phosphate dehydrogenase (GAPDH), hypoxanthine phosphoribosyltransferase-1 (HPRT1), and ribosomal protein lateral stalk subunit P0 (RPLP0)], and each $\mathrm{C}_{\mathrm{T}}$-value was normalized to the mean $C_{T}$-value of these genes as: $\Delta C_{T}=C_{T}$ gene of interest $-C_{T}$ mean of housekeeping genes. Normalized gene expression levels were calculated from three biological replicates of cultured cells or from three tumors as $2^{- \text {mean }}$ $\Delta \mathrm{CT}$.

\section{Statistical analysis}

The Spearman rank order test was used to search for correlations between parameters. Comparisons of survival curves were performed using the log-rank test. 
Comparisons of other data sets were conducted using the Student's $t$ test when the data complied with the conditions of normality and equal variance. Under other conditions, comparisons were carried out by non-parametric analysis using the Mann-Whitney rank sum test. Probability values of $P<0.05$ were considered significant. Statistical analysis was performed using the SigmaStat statistical software (SPSS, Chicago, IL).

\section{Results}

Bevacizumab treatment prolonged the survival of mice with meningeal A-07 tumors

Both melanoma models developed meningeal tumors after intracranial tumor cell inoculation (Fig. 1a). The meningeal tumors induced severe clinical symptoms and limited the survival of the host mice. The most frequent symptoms were weight loss, disconnection of scull sutures, destruction of scull bone, and extracranial a
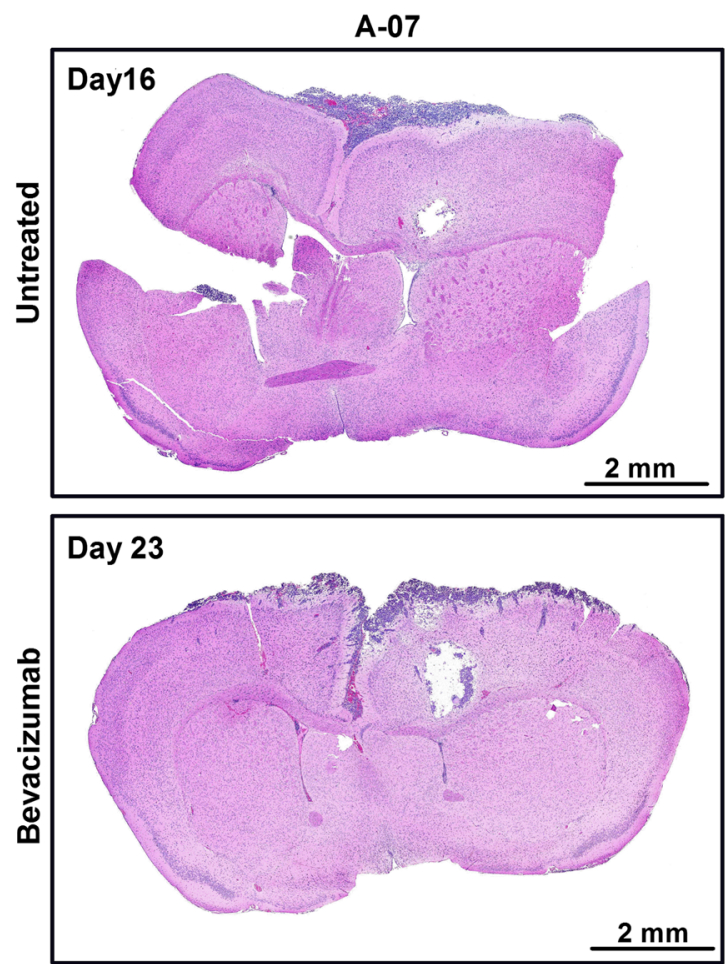

D-12
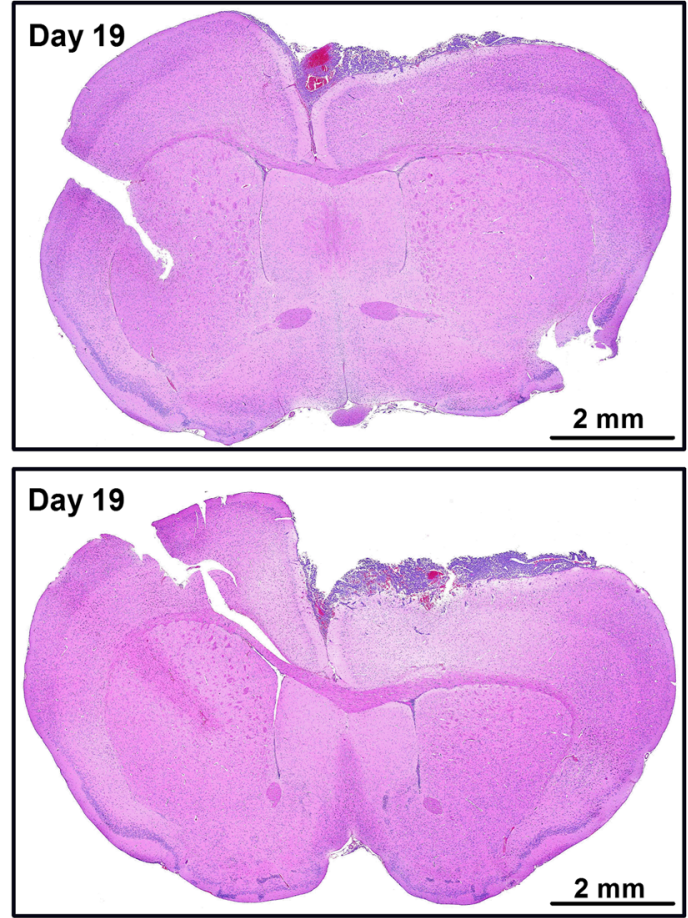

b
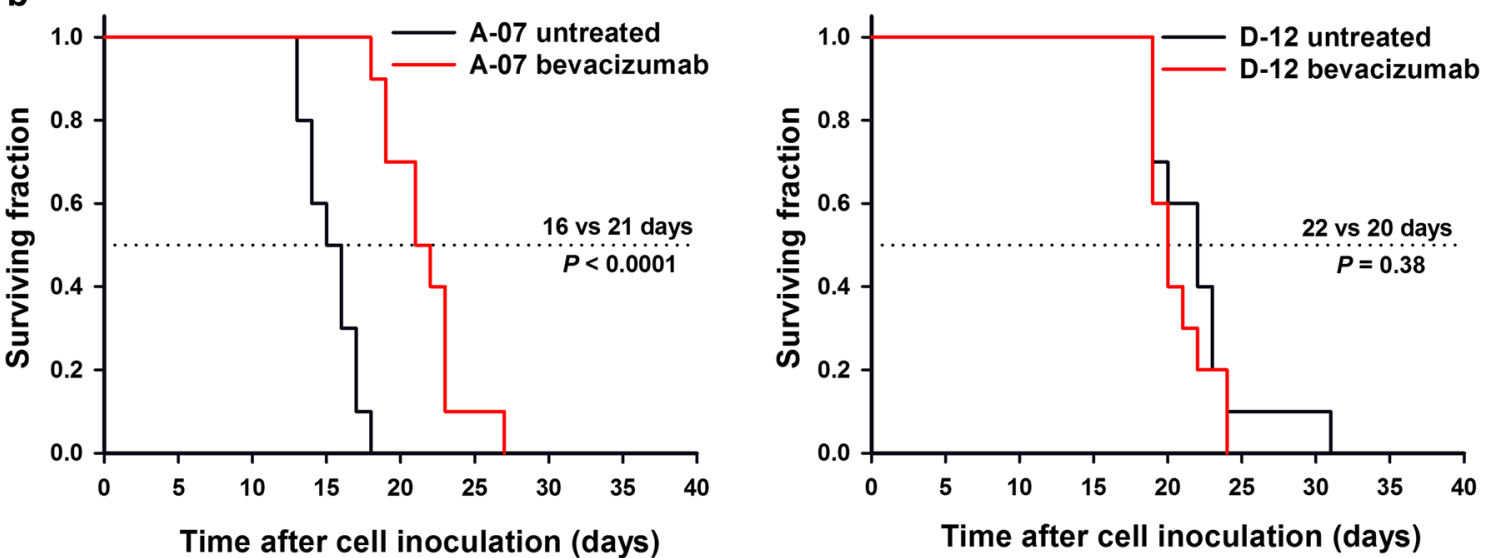

Fig. 1 Tumor growth and host survival. a Histological preparations of brains from untreated and bevacizumab-treated mice showing that A-07 and D-12 cells developed meningeal tumors after intracranial cell inoculation. The day numbers indicate time after cell inoculation. $\mathbf{b}$ Fraction of surviving mice versus time after cell inoculation for untreated and bevacizumab-treated mice with meningeal A-07 or D-12 tumors ( $N=10$ in each group). Bevacizumab treatment prolonged the survival of mice with A-07 tumors but not the survival of mice with D-12 tumors. $P$-values: log-rank test 
bleedings. The clinical symptoms and tumor growth pattern did not differ between bevacizumab-treated and untreated mice in any of the models. The median survival of untreated mice was 16 days (A-07) and 22 days (D-12). Bevacizumab treatment prolonged the median survival of mice with A-07 tumors to 21 days $(P<0.0001$; Fig. $1 \mathrm{~b})$, but did not prolong the median survival of mice with D-12 tumors $(P=0.38$; Fig. $1 b)$.

\section{Bevacizumab treatment inhibited angiogenesis in meningeal A-07 tumors}

Meningeal A-07 and D-12 tumors showed substantial angiogenic activity. CD31-stained histological preparations revealed highly vascularized tumors with enlarged and irregular blood vessels in both models, regardless of whether the tumors were treated with bevacizumab or not (Fig. 2a). MVD did not differ between bevacizumabtreated and untreated tumors in any of the models $[P=0.19$ (A-07) and $P=0.16$ (D-12); Fig. 2b]. The angiogenic activity was lower in bevacizumab-treated than in untreated tumors in the A-07 $(P=0.032)$ but not in the D-12 $(P=0.20)$ model (Fig. 2b), implying that bevacizumab inhibited angiogenesis in meningeal A-07 tumors but not in meningeal D-12 tumors.

\section{Bevacizumab-treated meningeal A-07 tumors showed increased cerebral invasion}

Multiple colonies of GFP-positive tumor cells were observed within the cerebral parenchyma of mice with meningeal tumors of both models (Fig. 3a). Most of these micrometastases were located close to the meningeal border, and evaluation of GFP-stained, HE-stained, and CD31-stained adjacent histological preparations revealed that the micrometastases grew around and along cerebral blood vessels (Fig. 3b). These vessels had a normal morphology, suggesting vessel co-option rather than sprouting angiogenesis.

Examination of GFP-stained histological sections from mice with A-07 tumors revealed that the density of micrometastases was higher in bevacizumab-treated than in untreated mice, and furthermore, the density and size of the micrometastases increased with the survival time of the host mouse. This is illustrated in Fig. 3a, showing the meningeal border of an untreated mouse with a survival time of 16 days and bevacizumab-treated mice with survival times of 18, 23, and 27 days. Furthermore, the border between the meningeal tumor and the cerebral parenchyma appeared increasingly more diffuse with increasing mouse survival, suggesting continuous cerebral invasion of melanoma cells (Fig. 3a). Quantitative analysis confirmed that the density of micrometastases was higher in bevacizumab-treated than in untreated A-07 mice ( $P=0.0050$; Fig. $3 \mathrm{c})$, and moreover, there was a strong positive correlation between the density of micrometastases and host survival time $(P<0.0001$; Fig. $3 \mathrm{c})$.

The morphological appearance of the micrometastases from D-12 meningeal tumors was similar to that of the A-07 micrometastases. However, the density of micrometastases was not significantly different in bevacizumab-treated and untreated $D-12$ mice $(P=0.15$; Fig. 3c). Moreover, there was no correlation between the density of micrometastases and the survival time of the host mouse $(P>0.05$; Fig. $3 \mathrm{c})$.

\section{Angiogenesis was driven by different pathways in meningeal A-07 and D-12 tumors}

To search for possible explanations of the differential responses to bevacizumab treatment of A-07 and D-12 tumors, the expression of angiogenesis-related genes in cultured cells and meningeal tumors was assessed by quantitative PCR. The expression of VEGF-A, the target of bevacizumab, was significantly higher in the A-07 model than in the D-12 model, both in cell cultures and meningeal tumors $(P<0.0001$ and $P=0.0014$, respectively; Fig. 4a). Of the 84 genes included in the PCR array, as much as $38 \%$ (cells) and $42 \%$ (tumors) showed more than fivefold higher expression in the D-12 model than in the A-07 model (Fig. 4b). On the other hand, only $11 \%$ (cells) and 5\% (tumors) of the genes showed more than fivefold higher expression in the A-07 model than in the D-12 model (Fig. 4b). The expression of the specific genes showing expression levels differing by a factor of more than 5 between meningeal A-07 and D-12 tumors is presented in Fig. 4c. Interestingly, the genes encoding angiopoietin-2 (ANGPT2) and interleukin-8 (IL8), two well known drivers of angiogenesis in melanoma, showed substantially higher expression (416-fold and 24-fold, respectively) in D-12 tumors than in A-07 tumors.

\section{Bevacizumab-treated meningeal A-07 tumors showed increased expression of angiogenesis-related genes}

To investigate whether bevacizumab treatment induced changes in the angiogenic profile of meningeal tumors, tumor tissue from bevacizumab-treated and untreated mice was subjected to quantitative PCR. In A-07 tumors, 17 of the 84 genes (20\%) showed a significant and more than twofold increase in expression after bevacizumab treatment (Fig. 5). These genes included ANGPT2 and its receptors TIE1 and TIE2, the matrix metalloproteases MMP9 and MMP14, and the genes encoding the two subunits of integrin $\alpha v \beta 3$ (ITGAV and ITGB3). In contrast, the expression levels in bevacizumab-treated and untreated D-12 tumors were not significantly different for any of the genes included in the analysis (Fig. 5). 

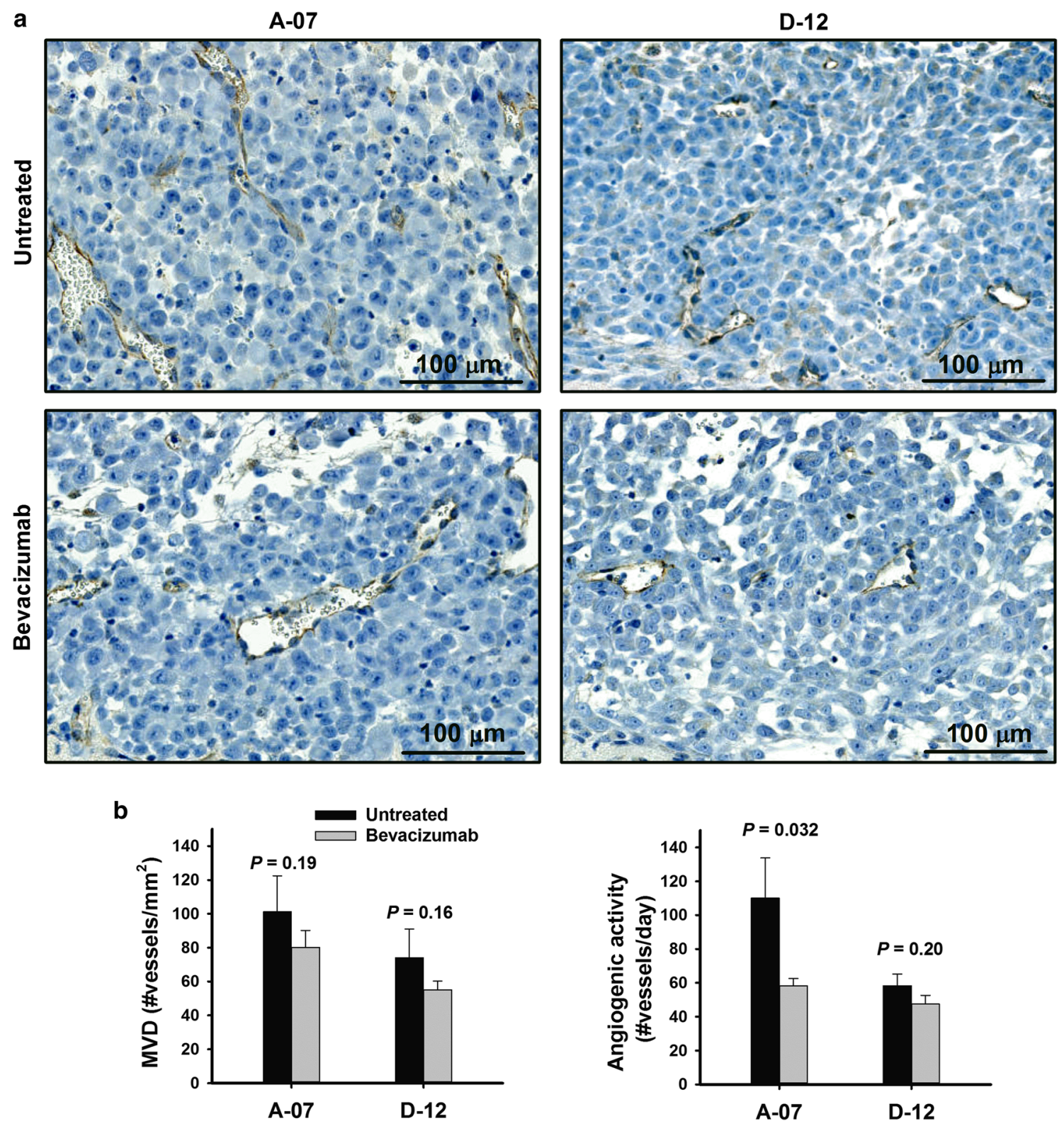

Tumor model

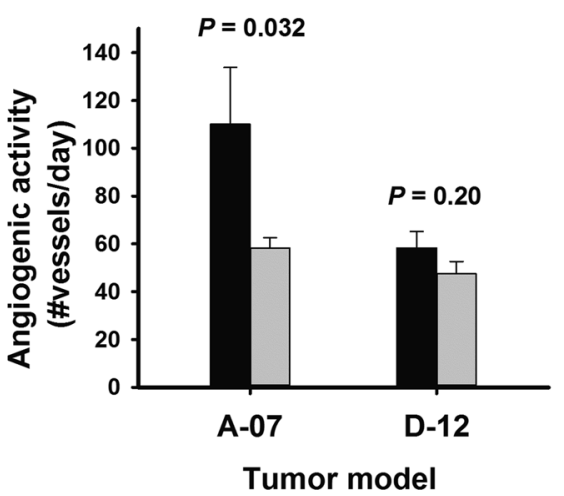

Fig. 2 Microvascular density and angiogenic activity. a CD31-stained histological preparations of untreated and bevacizumab-treated meningeal A-07 and D-12 tumors illustrating that the tumors were vascularized by abnormal vessels. b Microvascular density (MVD) and angiogenic activity in untreated and bevacizumab-treated meningeal A-07 and D-12 tumors. Bevacizumab treatment did not reduce MVD in any of the models, whereas angiogenic activity was reduced in bevacizumab-treated A-07 tumors but not in bevacizumab-treated D-12 tumors. Columns: mean of 5 tumors. Bars: standard error. $P$-values: one-sided t-test

\section{Discussion}

The present study showed that bevacizumab treatment prolonged the survival of mice bearing meningeal A-07 tumors, most likely because bevacizumab reduced the angiogenic activity and growth rate of the tumor tissue. These observations are in accordance with previous studies of intradermal A-07 tumors, having revealed a close link between angiogenic activity and tumor growth rate [29], reduced angiogenesis after treatment with an antiVEGF-A antibody [34], and reduced growth rate and vessel density after treatment with sunitinib, a tyrosine kinase inhibitor targeting the VEGF receptors (VEGFR) [35]. Moreover, our A-07 data are in agreement with clinical data suggesting that meningeal melanoma lesions are 


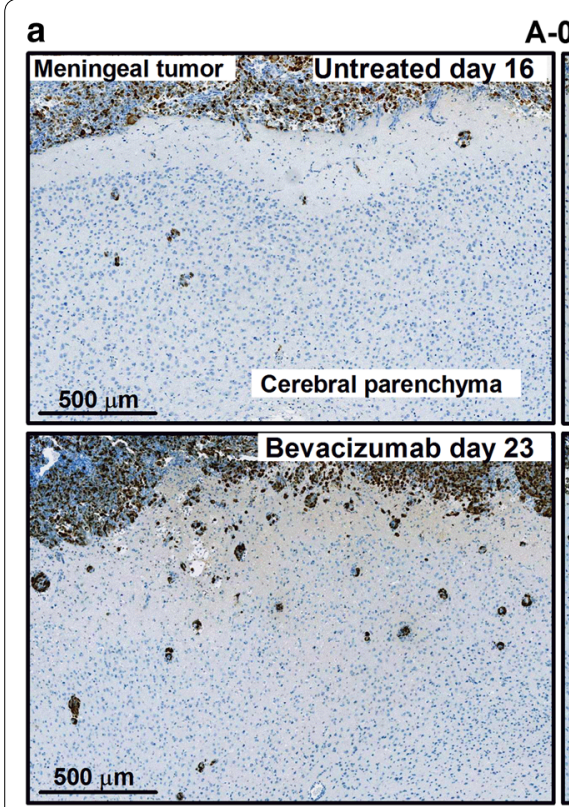

A-07
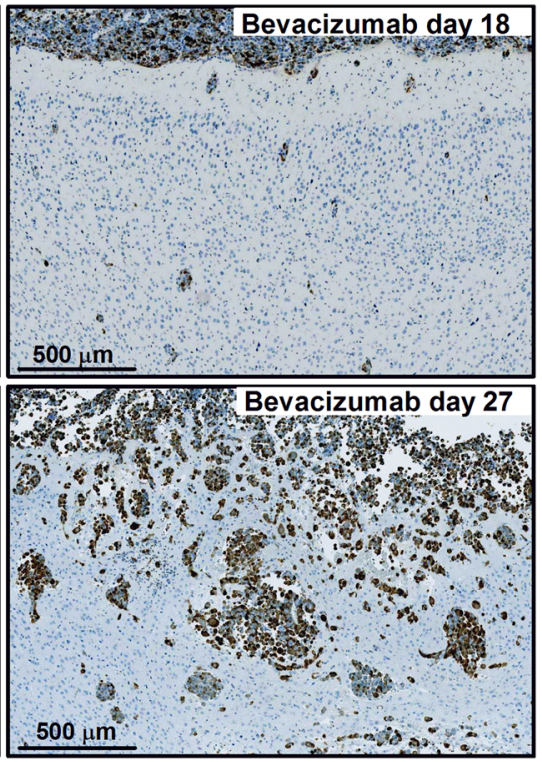

b

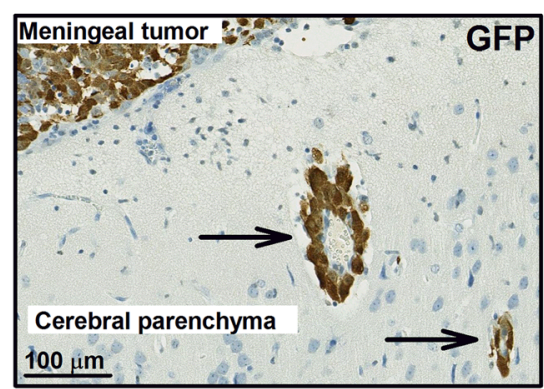

c

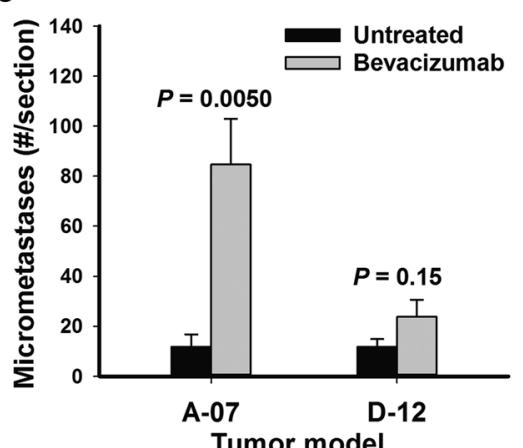

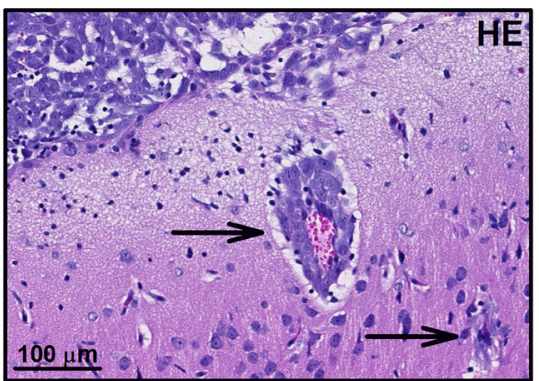

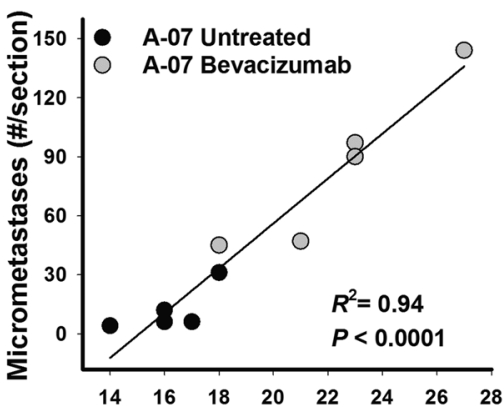

Survival time (days)
D-12
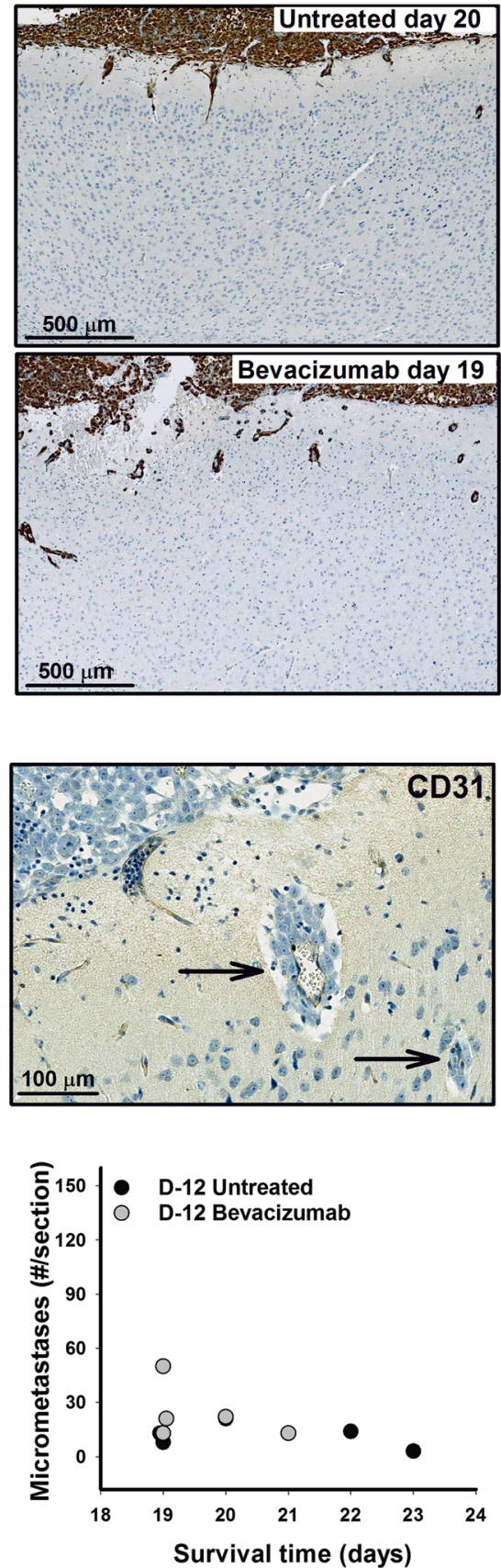

Fig. 3 Invasion of meningeal tumors into the cerebrum. a GFP-stained histological preparations of the meningeal-cerebral border in untreated and bevacizumab-treated mice showing that A-07 and D-12 meningeal tumors invaded the cerebral parenchyma and developed micrometastases. b Adjacent GFP-stained, HE-stained, and CD31-stained histological preparations showing two cerebral A-07 micrometastases (arrows) supplied by a central co-opted vessel. c The density of A-07 cerebral micrometastases was higher in bevacizumab-treated mice than in untreated mice and correlated with host survival time, whereas the density of D-12 cerebral micrometastases did not differ between untreated and bevacizumab-treated mice and was independent of host survival time. Columns: mean of 5 tumors. Bars: standard error. Points: individual mice. Curve: linear regression. P-values: two-sided t-test (left panel) or Spearman rank order test (middle panel) 

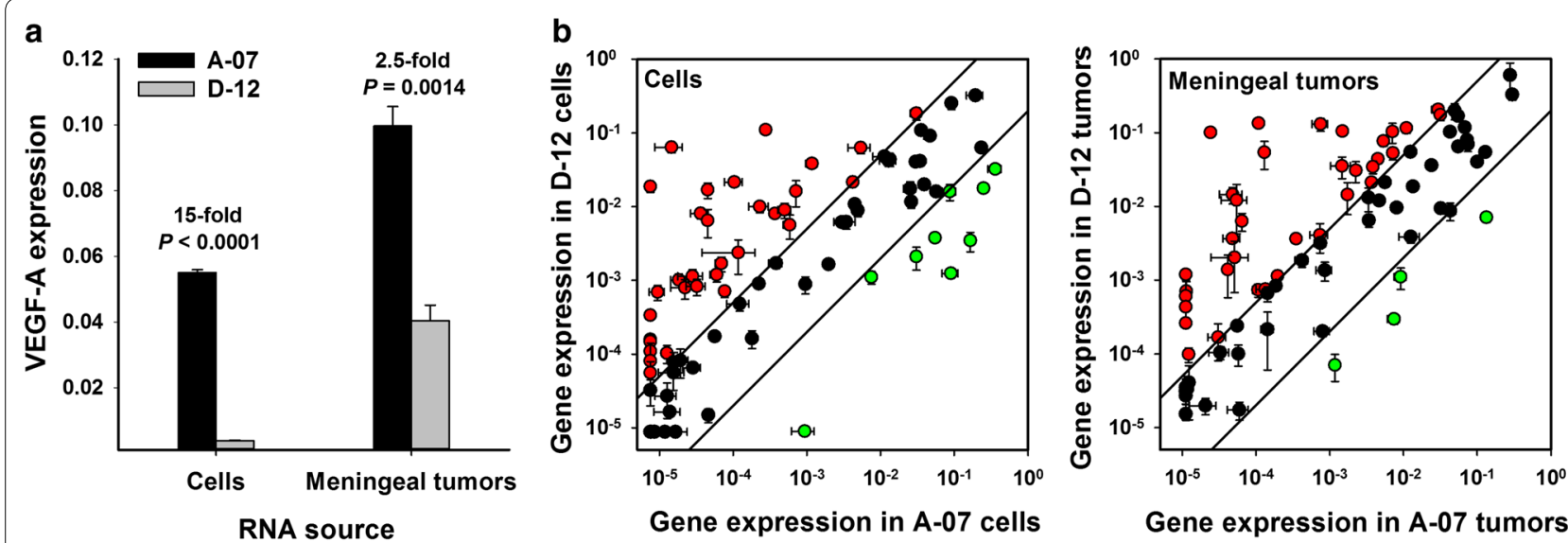

Gene expression in A-07 tumors

C

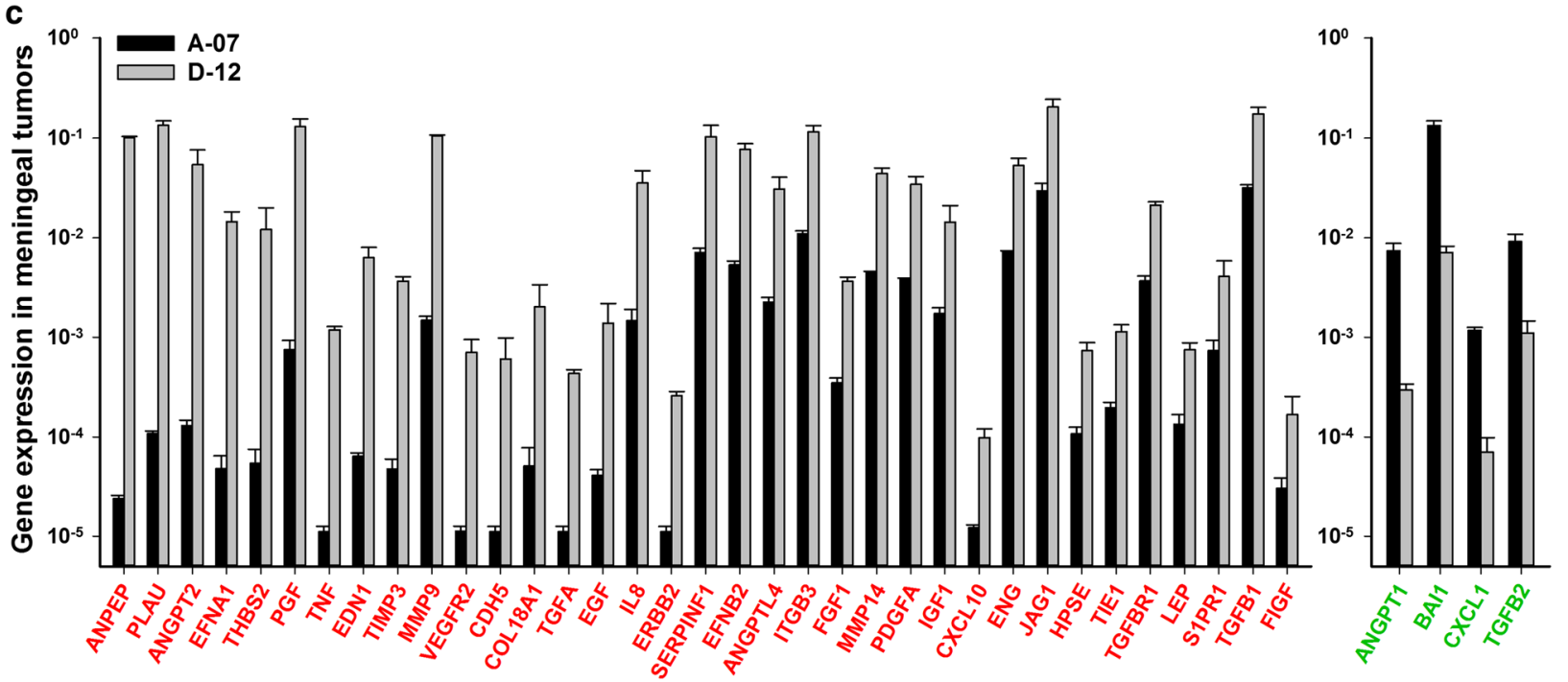

Angiogenesis-related gene

Fig. 4 Expression of angiogenesis-related genes. a The expression of VEGF-A was higher in the A-07 model than in the D-12 model, both in cultured cells and meningeal tumors. b The expression of 84 angiogenesis-related genes in the D-12 model versus that in the A-07 model in cultured cells and meningeal tumors. The lines indicate a fivefold difference in expression level. Of the 84 genes, 38\% (cells) and 42\% (tumors) showed more than fivefold higher expression in the D-12 model than in the A-07 model (red symbols), whereas 11\% (cells) and 5\% (tumors) showed more than fivefold higher expression in the A-07 model than in the D-12 model (green symbols). c The expression of the specific genes showing expression levels differing by a factor of more than 5 between meningeal A- 07 and D-12 tumors. Gene expression was measured with quantitative PCR and normalized to the expression of 5 housekeeping genes. Columns and points: mean of 3 cell cultures or tumors. Bars: standard error. $P$-values: two-sided $t$-test

susceptible to antiangiogenic therapy $[8,28]$, and with a preclinical study showing prolonged survival of mice with leptomeningeal metastases treated with the endogenous angiogenesis inhibitor angiostatin [24].

Although the survival of mice with meningeal A-07 tumors was prolonged after bevacizumab treatment, the treatment was not sufficient to block tumorinduced angiogenesis. We cannot exclude the possibility that the angiogenic activity of bevacizumab-treated meningeal tumors was a result of continued VEGF-A activity. Because bevacizumab is specific for human VEGF-A, any contribution from stromal-derived VEGF-A would not have been inhibited by bevacizumab [15]. Moreover, the bevacizumab dose may not have been sufficiently high to neutralize all tumor cell-derived VEGF-A. Low doses are however more clinically relevant in a preventive setting due to long treatment periods, and Ilhan-Mutlu et al. [21] have demonstrated prevention of brain metastasis formation in a lung adenocarcinoma xenograft model using a 

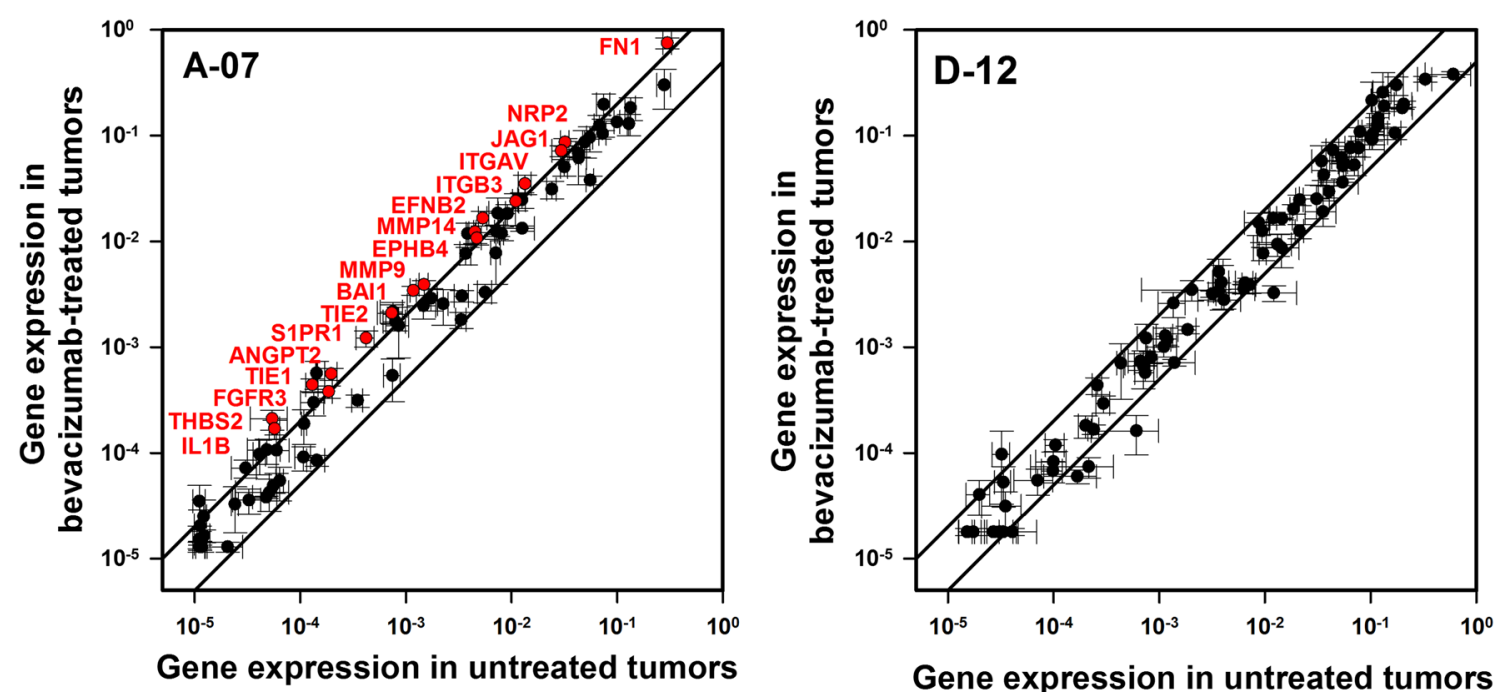

Fig. 5 Changes in the expression of angiogenesis-related genes after bevacizumab treatment. The expression of 84 angiogenesis-related genes in bevacizumab-treated versus that in untreated meningeal A-07 and D-12 tumors. The lines indicate a twofold difference in expression level. Of the 84 genes, 17 (20\%) showed a significant and more than twofold higher expression in bevacizumab-treated than in untreated A-07 tumors (red symbols), whereas none of the 84 genes showed expression levels that were significantly different in bevacizumab-treated and untreated D-12 tumors. Gene expression was measured with quantitative PCR and normalized to the expression of 5 housekeeping genes. Points: mean of 3 tumors. Bars: standard error

substantially lower dose of bevacizumab than that used in our study (2.5 versus $10 \mathrm{mg} / \mathrm{kg})$.

Despite a possible contribution from residual VEGFA activity, it is more likely that other angiogenic factors contributed to the sustained angiogenesis of bevacizumab-treated meningeal A-07 tumors. In addition to VEGF-A, the angiogenesis of intradermal A-07 tumors has been shown to depend on multiple angiogenic factors, including IL8, basic fibroblast growth factor, and platelet-derived endothelial cell growth factor [34]. The meningeal A-07 tumors expressed a large number of angiogenesis-related genes, including the genes encoding these factors. Furthermore, several angiogenesisrelated genes, including ANGPT2 and its receptors TIE1 and TIE2, were up-regulated after bevacizumab-treatment. Next to the VEGF/VEGFR pathway, the ANGPT/ TIE pathway is the most important angiogenic pathway in melanoma [14]. Thus, bevacizumab-treated meningeal tumors compensated for the loss of VEGF-A activity by up-regulating other angiogenesis-related genes and activating other angiogenic pathways. Consequently, combined inhibition of multiple angiogenic stimulators may be necessary to prevent angiogenesis in meningeal metastases even from VEGF-A-dependent melanomas like A-07.

In contrast to meningeal A-07 tumors, meningeal D-12 tumors did not show inhibited angiogenesis and growth rate after bevacizumab treatment. It is likely that this difference was a consequence of the differing angiogenic signature of the two melanoma models. Compared with A-07 tumors, D-12 tumors showed low expression of VEGF-A, but high expression of a large number of other angiogenic stimulators, including IL8, ANGPT2, urokinase plasminogen activator (PLAU), MMP9, and plateletderived growth factor A (PDGFA). These factors have been recognized as important drivers of melanoma angiogenesis [14], and may have contributed to the bevacizumab-resistant angiogenesis of meningeal D-12 tumors. Interestingly, previous studies have revealed that IL8 and ANGPT2 play important roles in the angiogenesis and spontaneous pulmonary metastasis of intradermal D-12 tumors $[34,36]$.

Importantly, intradermal D-12 tumors have also been shown to be sensitive to anti-VEGF-A treatment, since tumor angiogenesis and the development of pulmonary metastases were reduced significantly in tumor-bearing mice treated with a neutralizing antibody against VEGFA [34]. The present study taken together with that study suggests that the susceptibility of melanoma metastases to VEGF-A-targeting treatments may differ among metastatic sites. Consequently, efficient antiangiogenic treatment of melanoma patients with metastatic growth in more than a single organ may require the use of inhibitors of multiple angiogenic pathways.

Tumor cells of both melanoma models were seen to migrate from meningeal tumors into the 
cerebral parenchyma where they formed micrometastases. Whereas the growth of the meningeal metastases depended on sprouting angiogenesis, the growth of the cerebral metastases depended on vessel co-option. This difference in vascularization was probably a consequence of differences between meningeal and cerebral blood vessel in their susceptibility to angiogenic factors secreted by melanoma cells $[27,37]$.

The growth of meningeal A-07 metastases was inhibited by bevacizumab treatment but not the growth of cerebral A-07 metastases, probably because of the distinctly different mode of vascularization. Vessel co-option has also been seen previously to infer resistance to antiangiogenic therapy in preclinical models of melanoma brain metastases [22, 23]. Kienast et al. [23] used a cranial window chamber model to show that bevacizumab inhibited angiogenesis and induced dormancy of metastases established from lung carcinoma cells but had no effect on melanoma metastases showing a co-optive growth pattern. Moreover, Leenders et al. [22] established cerebral melanoma metastases in mice by intracarotid artery injection of tumor cells and showed that antiangiogenic therapy with a VEGF targeting agent resulted in sustained tumor progression via vessel co-option.

In the present study, bevacizumab appeared to promote cerebral invasion of meningeal A-07 cells and increase the development of micrometastases rather than inhibit metastatic growth in the cerebral parenchyma. The brains were removed for histological examination when the host mice were moribund, and the observed increase in cerebral invasion and metastatic growth was most likely a consequence of the increased survival time of bevacizumab-treated mice, allowing the micrometastases more time to develop in treated than in untreated mice. This suggestion is supported by the observation that the density of micrometastases correlated strongly with mouse survival time. Furthermore, the size of the micrometastases appeared to increase with the density of cerebral lesions.

However, there is evidence that antiangiogenic therapy can increase the invasive and metastatic potential of tumor cells $[38,39]$. The bevacizumab-treated meningeal A-07 tumors showed increased expression of a large number of genes, including MMP9, MMP14, and those encoding the two subunits of integrin $\alpha_{v} \beta_{3}$. MMPs are proteolytic enzymes that degrade some components of the extracellular matrix, whereas integrins mediate cellcell and cell-extracellular matrix interactions, and both protein families are associated with cancer-cell invasion and metastasis [40, 41]. MMP9 has been shown to enhance the invasiveness of A-07 cells in vitro [42] and to facilitate the migration of leukocytes across the membrane separating pia mater from the cerebral cortex [43]. We can therefore not exclude that bevacizumab treatment increased the cerebral invasion of meningeal A-07 tumors by up-regulating genes promoting tumor cell migration, a possibility that warrants further investigations.

While the prognosis of patients diagnosed with melanoma brain metastases remains poor, the incidence is expected to increase both as a result of a general increase in melanoma incidence and as a result of prolonged patient survival due to improved control of extracranial disease [44]. Novel strategies for the treatment of melanoma patients with brain involvement are therefore highly warranted. A great advantage of antiangiogenic drugs such as bevacizumab is that they target endothelial cells and can exert their effect without crossing the blood-brain barrier [21]. However, despite the important role of angiogenesis in melanoma progression and metastasis, the data reported here suggest that the development of antiangiogenic strategies for the treatment of melanoma brain metastases may be highly challenging as the outcome of treatment may depend on the intracranial site of metastatic growth as well as the angiogenic signature of the melanoma cells and the extent of treatmentinduced changes in this signature.

\section{Conclusions}

Bevacizumab treatment inhibited tumor angiogenesis and prolonged tumor host survival in mice with meningeal A-07 tumors, but had no effect on the angiogenic activity of meningeal D-12 tumors. Meningeal A-07 tumors compensated for reduced VEGF-A-mediated angiogenic activity by up-regulating the expression of a large number of other angiogenesis-related genes, including genes governing the ANGPT/TIE pathway. Melanoma cells migrated from meningeal tumors into the cerebral parenchyma and formed micrometastases vascularized by vessel co-option, and in the A- 07 model, bevacizumab-treated mice developed more and larger cerebral micrometastases than untreated mice. These discoveries suggest that antiangiogenic therapy may have the potential to inhibit the growth of meningeal melanoma metastases, but emphasize the need to target multiple angiogenic pathways and to individualize the treatment based on the angiogenic signature of the tumor tissue. Furthermore, antiangiogenic therapy cannot be expected to improve the outcome of meningeal melanoma metastases without being combined with therapeutic strategies for preventing tumor cell migration, vessel co-option, and metastatic growth in the cerebral parenchyma. 


\section{Abbreviations}

ACTB: $\beta$-actin; ANGPT2: angiopoietin-2; BALB/c: Bagg albino/c; B2M: $\beta-2$ microglobulin; BRAF: B-Raf proto-oncogene; GAPDH: glyceraldehyde-3-phosphate dehydrogenase; GFP: green fluorescence protein; HBSS: Hanks' balanced salt solution; HE: hematoxylin and eosin; HEPES: 4-(2-hydroxyethyl)-1-piperazineethanesulfonic acid; HPRT1: hypoxanthine phosphoribosyltransferase-1; IL8: interleukin-8; ITGAV: integrin a-V; ITGB3: integrin $\beta$-3; MMP9 and 14: matrix metalloprotease 9 and 14; MRI: magnetic resonance imaging; MVD: microvascular density; PCR: polymerase chain reaction; RPLPO: ribosomal protein lateral stalk subunit P0; PLAU: urokinase plasminogen activator; PDGFA: plateletderived growth factor-A; TIE1 and 2: tyrosine kinase with immunoglobulin-like and epidermal growth factor-like domains 1 and 2; VEGF-A: vascular endothelial growth factor-A; VEGFR: vascular endothelial growth factor receptor.

\section{Acknowledgements}

Not applicable.

\section{Authors' contributions}

TGS and EKR conceived the study, designed the experiments, performed the statistical analyses, and wrote the manuscript. TGS and JVG carried out the experiments. TGS, JVG, and EKR analyzed and interpreted the experimental data. All authors read and approved the final manuscript.

\section{Funding}

This study was supported by the Norwegian Cancer Society and the SouthEastern Norway Regional Health Authority. The funding bodies were not involved in the design of the study and collection, analysis, and interpretation of data and in writing the manuscript. Funding was provided by Kreftforeningen (Grant No. 205217), and Helse Sør-Øst RHF (Grant No. 2017080).

\section{Availability of data and materials}

The datasets used and analyzed during the current study are available from the corresponding author on reasonable request.

\section{Ethics approval and consent to participate}

Animal experiments were approved by the Institutional Committee on Research Animal Care, Department of Comparative Medicine, Oslo University Hospital, Norway and the Norwegian Food Safety Authority, Brummundal, Norway (approved protocol: FOTS ID 10422). The use of human tissue for establishing the melanoma models was approved by our institution (Committee for Medical and Health Research Ethics).

\section{Consent for publication}

Not applicable.

\section{Competing interests}

The authors declare that they have no competing interests.

Received: 30 September 2019 Accepted: 3 January 2020

Published online: 08 January 2020

\section{References}

1. Westphal D, Glitza Oliva IC, Niessner H. Molecular insights into melanoma brain metastases. Cancer. 2017;123(Suppl 11):2163-75.

2. Davies MA, Liu P, McIntyre S, Kim KB, Papadopoulos N, Hwu WJ, et al. Prognostic factors for survival in melanoma patients with brain metastases. Cancer. 2011;117:1687-96.

3. Amer MH, Al-Sarraf M, Baker LH, Vaitkevicius VK. Malignant melanoma and central nervous system metastases: incidence, diagnosis, treatment and survival. Cancer. 1978;42:660-8.

4. Fonkem E, Uhlmann EJ, Floyd SR, Mahadevan A, Kasper E, Eton O, et al. Melanoma brain metastasis: overview of current management and emerging targeted therapies. Expert Rev Neurother. 2012;12:1207-15.

5. Raizer JJ, Hwu WJ, Panageas KS, Wilton A, Baldwin DE, Bailey E, et al. Brain and leptomeningeal metastases from cutaneous melanoma: survival outcomes based on clinical features. Neuro Oncol. 2008;10:199-207.

6. Cohen JV, Tawbi H, Margolin KA, Amravadi R, Bosenberg M, Brastianos PK, et al. Melanoma central nervous system metastases: current approaches, challenges, and opportunities. Pigment Cell Melanoma Res. 2016;29:627-42.

7. Chamberlain MC, Baik CS, Gadi VK, Bhatia S, Chow LQM. Systemic therapy of brain metastases: non-small cell lung cancer, breast cancer, and melanoma. Neuro Oncol. 2017;19:i1-24.

8. Chamberlain MC. Leptomeningeal metastasis. Curr Opin Oncol. 2010;22:627-35.

9. Lo JA, Fisher DE. The melanoma revolution: from UV carcinogenesis to a new era in therapeutics. Science. 2014;346:945-9.

10. Margolin K. The promise of molecularly targeted and immunotherapy for advanced melanoma. Curr Treat Options Oncol. 2016;17:48.

11. Berghoff AS, Preusser M. Targeted therapies for melanoma brain metastases. Curr Treat Options Neurol. 2017;19:13.

12. Patel JK, Didolkar MS, Pickren JW, Moore RH. Metastatic pattern of malignant melanoma. A study of 216 autopsy cases. Am J Surg. 1978;135:807-10

13. Steeg PS, Camphausen KA, Smith QR. Brain metastases as preventive and therapeutic targets. Nat Rev Cancer. 2011;11:352-63.

14. Jour G, Ivan D, Aung PP. Angiogenesis in melanoma: an update with a focus on current targeted therapies. J Clin Pathol. 2016;69:472-83.

15. Ferrara N, Hillan KJ, Gerber HP, Novotny W. Discovery and development of bevacizumab, an anti-VEGF antibody for treating cancer. Nat Rev Drug Discov. 2004;3:391-400.

16. Corrie PG, Marshall A, Dunn JA, Middleton MR, Nathan PD, Gore M, et al. Adjuvant bevacizumab in patients with melanoma at high risk of recurrence (AVAST-M): preplanned interim results from a multicentre, open-label, randomised controlled phase 3 study. Lancet Oncol. 2014;15:620-30.

17. Kusters B, Leenders WP, Wesseling P, Smits D, Verrijp K, Ruiter DJ, et al. Vascular endothelial growth factor-A(165) induces progression of melanoma brain metastases without induction of sprouting angiogenesis. Cancer Res. 2002;62:341-5.

18. Kusters B, de Waal RM, Wesseling P, Verrijp K, Maass C, Heerschap A, et al. Differential effects of vascular endothelial growth factor $A$ isoforms in a mouse brain metastasis model of human melanoma. Cancer Res. 2003;63:5408-13.

19. Oku T, Tjuvajev JG, Miyagawa T, Sasajima T, Joshi A, Joshi R, et al. Tumor growth modulation by sense and antisense vascular endothelial growth factor gene expression: effects on angiogenesis, vascular permeability, blood volume, blood flow, fluorodeoxyglucose uptake, and proliferation of human melanoma intracerebral xenografts. Cancer Res. 1998:58:4185-92.

20. Xie TX, Huang FJ, Aldape KD, Kang SH, Liu M, Gershenwald JE, et al. Activation of stat 3 in human melanoma promotes brain metastasis. Cancer Res. 2006;66:3188-96

21. Ilhan-Mutlu A, Osswald M, Liao YX, Gommel M, Reck M, Miles D, et al. Bevacizumab prevents brain metastases formation in lung adenocarcinoma. Mol Cancer Ther. 2016;15:702-10.

22. Leenders WP, Kusters B, Verrijp K, Maass C, Wesseling P, Heerschap A, et al. Antiangiogenic therapy of cerebral melanoma metastases results in sustained tumor progression via vessel co-option. Clin Cancer Res. 2004;10(18 Pt 1):6222-30.

23. Kienast $Y$, von Baumgarten $L$, Fuhrmann $M$, Klinkert WE, Goldbrunner $R$, Herms J, et al. Real-time imaging reveals the single steps of brain metastasis formation. Nat Med. 2010;16:116-22.

24. Reijneveld JC, Taphoorn MJB, Kerckhaert OAJ, Drixler TA, Boogerd W, Voest EE. Angiostatin prolongs the survival of mice with leptomeningeal metastases. Eur J Clin Investig. 2003;33:76-81.

25. Berghoff AS, Rajky O, Winkler F, Bartsch R, Furtner J, Hainfellner JA, et al. Invasion patterns in brain metastases of solid cancers. Neuro Oncol. 2013;15:1664-72.

26. Hung T, Morin J, Munday WR, Mackenzie IR, Lugassy C, Barnhill RL. Angiotropism in primary cutaneous melanoma with brain metastasis: a study of 20 cases. Am J Dermatopathol. 2013;35:650-4.

27. Simonsen TG, Gaustad JV, Rofstad EK. Intertumor heterogeneity in vascularity and invasiveness of artificial melanoma brain metastases. J Exp Clin Cancer Res. 2015;34:150.

28. Herrlinger $U$, Wiendl $H$, Renninger $M$, Forschler H, Dichgans J, Weller M. Vascular endothelial growth factor (VEGF) in leptomeningeal metastasis: diagnostic and prognostic value. Br J Cancer. 2004;91:219-24. 
29. Rofstad EK. Orthotopic human melanoma xenograft model systems for studies of tumor angiogenesis, pathophysiology, treatment sensitivity and metastatic pattern. Br J Cancer. 1994;70:804-12.

30. Rofstad EK, Måseide K. Radiobiological and immunohistochemical assessment of hypoxia in human melanoma xenografts: acute and chronic hypoxia in individual tumours. Int J Radiat Biol. 1999;75:1377-93.

31. Graff BA, Bjørnæs I, Rofstad EK. Microvascular permeability of human melanoma xenografts to macromolecules: relationships to tumor volumetric growth rate, tumor angiogenesis, and VEGF expression. Microvasc Res. 2001;61:187-98.

32. Simonsen TG, Gaustad JV, Leinaas MN, Rofstad EK. High interstitial fluid pressure is associated with tumor-line specific vascular abnormalities in human melanoma xenografts. PLoS ONE. 2012;7:e40006.

33. VanGuilder HD, Vrana KE, Freeman WM. Twenty-five years of quantitative PCR for gene expression analysis. Biotechniques. 2008;44:619-26.

34. Rofstad EK, Halsør EF. Vascular endothelial growth factor, interleukin 8, platelet-derived endothelial cell growth factor, and basic fibroblast growth factor promote angiogenesis and metastasis in human melanoma xenografts. Cancer Res. 2000;60:4932-8.

35. Gaustad JV, Simonsen TG, Leinaas MN, Rofstad EK. Sunitinib treatment does not improve blood supply but induces hypoxia in human melanoma xenografts. BMC Cancer. 2012;12:388.

36. Huang R, Andersen LMK, Rofstad EK. Metastatic pathway and the microvascular and physicochemical microenvironments of human melanoma xenografts. J Transl Med. 2017;15:203.

37. Schackert G, Price JE, Zhang RD, Bucana CD, Itoh K, Fidler IJ. Regional growth of different human melanomas as metastases in the brain of nude mice. Am J Pathol. 1990;136:95-102.
38. Paez-Ribes M, Allen E, Hudock J, Takeda T, Okuyama H, Vinals F, et al. Antiangiogenic therapy elicits malignant progression of tumors to increased local invasion and distant metastasis. Cancer Cell. 2009;15:220-31.

39. Ebos JML, Lee CR, Cruz-Munoz W, Bjarnason GA, Christensen JG, Kerbel RS. Accelerated metastasis after short-term treatment with a potent inhibitor of tumor angiogenesis. Cancer Cell. 2009;15:232-9.

40. Egeblad M, Werb Z. New functions for the matrix metalloproteinases in cancer progression. Nat Rev Cancer. 2002;2:161-74.

41. Huang R, Rofstad EK. Integrins as therapeutic targets in the organ-specific metastasis of human malignant melanoma. J Exp Clin Cancer Res. 2018;37:92.

42. Rofstad EK, Mathiesen B, Kindem K, Galappathi K. Acidic extracellular pH promotes experimental metastasis of human melanoma cells in athymic nude mice. Cancer Res. 2006;66:6699-707.

43. Owens T, Bechmann I, Engelhardt B. Perivascular spaces and the two steps to neuroinflammation. J Neuropathol Exp Neurol. 2008;67:1113-21.

44. Fidler IJ. The biology of brain metastasis: challenges for therapy. Cancer J. 2015;21:284-93.

\section{Publisher's Note}

Springer Nature remains neutral with regard to jurisdictional claims in published maps and institutional affiliations.
Ready to submit your research? Choose BMC and benefit from:

- fast, convenient online submission

- thorough peer review by experienced researchers in your field

- rapid publication on acceptance

- support for research data, including large and complex data types

- gold Open Access which fosters wider collaboration and increased citations

- maximum visibility for your research: over $100 \mathrm{M}$ website views per year

At BMC, research is always in progress.

Learn more biomedcentral.com/submissions 